\title{
Significance of dynamic changes in gastric smooth muscle cell apoptosis, PI3K-AKT-mTOR and AMPK-mTOR signaling in a rat model of diabetic gastroparesis
}

\author{
MO-HAN ZHANG, JING-ZHI JIANG, YING-LAN CAI, LI-HUA PIAO and ZHENG JIN \\ Department of Histology and Embryology, Yanbian University College of Medicine, Yanji, Jilin 133002, P.R. China
}

Received June 17, 2016; Accepted April 19, 2017

DOI: $10.3892 / \mathrm{mmr} .2017 .6764$

\begin{abstract}
The aim of the present study was to investigate the significance of cell apoptosis, the phosphoinositide-3-kinase (PI3K)-protein kinase B (AKT)-mammalian target of rapamycin (mTOR) pathway, and the 5' adenosine monophosphate-activated protein kinase (AMPK)-mTOR pathways in the process of diabetic gastroparesis. Changes in gastric smooth muscle cells of diabetic rats with induced gastroparesis were examined. The diabetic rat model was established by dividing animals into a normal control group and diabetic model groups examined at 2, 4 and 6 weeks. Diabetic gastroparesis was evaluated by examining the rates of gastric residual pigment, whereas flow cytometry was used to detect the apoptosis of gastric smooth muscle cells. The expression levels of PI3K and
\end{abstract}

Correspondence to: Dr Zheng Jin, Department of Histology and Embryology, Yanbian University College of Medicine, 977 Gongyuan Road, Yanji, Jilin 133002, P.R. China

E-mail: jinzheng@ybu.edu.cn

Abbreviations: 4E-BP1, eukaryotic translation initiation factor 4-binding protein 1; AMP, adenosine monophosphate; AMPK, 5' adenosine monophosphate-activated protein kinase; AKT, protein kinase B; ATP, adenosine triphosphate; DM2W, diabetic model at 2 weeks; DM4W, diabetic model at 4 weeks; DM6W, diabetic model at 6 weeks; DM8W, diabetic model at 8 weeks; DMEM, Dulbecco's modified Eagle's medium; eIF4E, eukaryotic translation initiation factor 4E; mTOR, mammalian target of rapamycin; OD, optical density; NC, normal control; P-4E-BP1, phosphorylated eukaryotic translation initiation factor 4-binding protein 1; p-AKT, phosphorylated protein kinase B; p-AMPK, phosphorylated 5'-adenosine monophosphate-activated protein kinase; p-TSC-2, phosphorylated tuberous sclerosis complex 2; p-mTOR, phosphorylated mammalian target of rapamycin; p-p70S6K, phosphorylated p70 ribosomal S6 kinase; p70S6K, p70 ribosomal S6 kinase; PI, propidium iodide; PI3K, phosphoinositide-3-kinase; PVDF, polyvinylidene difluoride; STZ, streptozotocin; TSC-2, tuberous sclerosis complex 2

Key words: diabetic, gastroparesis, apoptosis, phosphoinositide-3kinase-protein kinase B-mammalian target of rapamycin, 5' adenosine monophosphate-activated protein kinase-mammalian target of rapamycin phosphorylated (p-) AKT, AMPK, mTOR, tuberous sclerosis complex 2, p70 ribosomal S6 kinase, and eukaryotic translation initiation factor 4-binding protein 1 were determined in gastric muscles using western blot analysis. Diabetic gastroparesis was confirmed in models at 6 weeks. The apoptosis of gastric smooth muscle cells gradually increased in all diabetic groups, and significant changes were observed in key proteins involved in PI3K-AKT-mTOR and AMPK-mTOR signaling. The results indicated that apoptosis was important in the occurrence of diabetic gastroparesis, and the PI3K-AKT-mTOR and AMPK-mTOR pathways were activated during the apoptotic processes, but were incapable of regulating apoptosis.

\section{Introduction}

Gastroparesis is one of the common chronic complications caused by diabetes mellitus, which primarily manifests as bloating, nausea and vomiting, and results in low gastric motility, delayed gastric emptying and prolonged gastric transit time (1). Although the apoptosis of gastric smooth muscle cells has been shown to be important in the occurrence of diabetic gastroparesis $(2,3)$, the mechanisms upstream of this process remain to be elucidated. The phosphoinositide-3-kinase (PI3K)-protein kinase B (AKT)-mammalian target of rapamycin (mTOR) pathway is an important intracellular signaling cascade capable of affecting a variety of cell behaviors, including cell proliferation, growth, apoptosis and metabolism $(4,5)$. PI3K phosphorylates phosphatidylinositol 4,5-bisphosphate into phosphatidylinositol 3,4,5-triphosphate to recruit and activate AKT, which subsequently activates its downstream target molecule, mTOR. mTOR is also a downstream target of 5 ' adenosine monophosphate-activated protein kinase (AMPK), which is widely involved in mediating cell metabolism, and has important biological roles in regulating cell apoptosis, physiological and pathological processes (6). AMPK phosphorylates and activates tuberous sclerosis complex 2 (TSC-2) upstream of mTOR to promote the formation of a TSC-1/TSC-2 complex, which is capable of inhibiting the activity of another upstream guanosine triphosphate-binding protein, Ras homolog enriched in brain, ultimately reducing the activity of mTOR $(7,8)$.

mTOR downstream targets include ribosomal S6 protein kinase (S6K) and eukaryotic translation initiation factor 4-binding protein 1 (4E-BP1) $(9,10)$. The phosphorylation 
of mTOR has been shown to activate the p70 form of S6K (p70S6K) and inhibit the binding of 4E-BP1 to eukaryotic translation initiation factor $4 \mathrm{E}$ (eIF4E), thereby releasing eIF4E and improving the translation of anti-apoptotic proteins (11).

However, the correlation between cell apoptosis, and the PI3K-AKT-mTOR and AMPK-mTOR pathways in diabetic gastroparesis has not been reported. In the present study, a rat model of diabetic gastroparesis was established to examine the apoptosis of gastric smooth muscle cells, and the key proteins involved in PI3K-AKT-mTOR and AMPK-mTOR signaling, including PI3K and phosphorylated forms of AKT (p-AKT), AMPK (p-AMPK), TSC-2 (p-TSC-2), mTOR (p-mTOR), p70S6k (p-p70S6K) and 4E-BP1 (P-4E-BP1). The aim of the present study was to elucidate the pathogenesis of diabetic gastroparesis to provide a scientific theoretical basis and experimental evidence for novel clinical treatments.

\section{Materials and methods}

Experimentalanimals. A total of 40 adult male Sprague-Dawley rats, weighing 200 $20 \mathrm{~g}$, were provided by Yanbian University Experimental Animal Center (Yanji, China). The rats were housed at room temperature $\left(18-25^{\circ} \mathrm{C}\right)$, with $50-80 \%$ relative humidity and a 12-h light/dark cycle, and allowed free access to food and water. The study was approved by the ethics committee of Yanbian University College of Medicine (Yanji, China).

Preparation of the diabetic model and experimental groups. Streptozotocin (STZ) solution (0.5\%; Sigma-Aldrich; Merck KGaA, Darmstadt, Germany) was prepared using citrate buffer ( $\mathrm{pH} 4.0 ; 0.1 \mathrm{~mol} / \mathrm{l})$. Following 1 week of adaptive feeding, the rats were deprived of food for $12 \mathrm{~h}$ with access to water, and received an intraperitoneal injection of $65 \mathrm{mg} / \mathrm{kg}$ STZ to establish the diabetic model. The breeding conditions remained unchanged. At 7 days post-injection, tail vein blood was collected and the success of the model was determined based on a glucose concentration $>350 \mathrm{mg} / \mathrm{dL}(12,13)$. The model rats were randomly divided into groups of 10 , including a normal control (NC) group and diabetic model (DM) groups examined 2 weeks (DM2W), 4 weeks (DM4W) and 6 weeks (DM6W) later.

Preparation of diabetic gastroparesis model. The rates of gastric residual pigment were detected, as they reflect the capacity for gastric emptying in the animal. Following $24 \mathrm{~h}$ of fasting, the rats were administered with $0.4 \mathrm{ml}$ of $1 \mathrm{mg} / \mathrm{ml}$ methylene blue solution and sacrificed by cervical dislocation 30 min later. The whole stomach was immediately harvested, and gastric residue was collected by removing the stomach mucosal layer and collecting the antral circular muscle strips, which were placed in liquid nitrogen. The residues were rinsed with saline and centrifuged at $744 \mathrm{x}$ g for $15 \mathrm{~min}$ at $4^{\circ} \mathrm{C}$, following which the supernatant was collected and optical density (OD) was detected at $640 \mathrm{~nm}$ using a spectrophotometer. The gastric residual pigment ratio was calculated as: OD value of detection tube/OD value of standard tube $\times 100 \%$. In terms of comparing the diabetic model groups with the NC group, $\mathrm{P}<0.05$ was considered to indicate successful diabetic gastroparesis model establishment (14).
Table I. Comparison of gastric residual pigment ratio and apoptotic rates.

\begin{tabular}{lcc}
\hline Group & $\begin{array}{c}\text { Gastric residual } \\
\text { pigment ratio }(\%)\end{array}$ & $\begin{array}{c}\text { Apoptotic } \\
\text { rate }(\%)\end{array}$ \\
\hline NC & $40.24 \pm 2.15$ & - \\
DM2W & $42.82 \pm 3.19$ & $1.54 \pm 0.46$ \\
DM4W & $44.72 \pm 3.10$ & $2.75 \pm 0.54$ \\
DM6W & $50.65 \pm 3.31^{\mathrm{a}}$ & $7.48 \pm 0.36^{\mathrm{b}}$ \\
\hline
\end{tabular}

Data are presented as the mean \pm standard deviation $(\mathrm{n}=10)$. ${ }^{\mathrm{a}} \mathrm{P}<0.05$, vs. NC group; ${ }^{\mathrm{P}} \mathrm{P}<0.05$, vs. $\mathrm{DM} 4 \mathrm{~W}$ group. $\mathrm{NC}$, normal control; $\mathrm{DM} 2 \mathrm{~W}$, diabetic model at 2 weeks; DM4W, diabetic model at 4 weeks; diabetic model at 6 weeks.

Preparation of gastric smooth muscle cell suspensions. To prepare cell suspensions, gastric smooth muscle was mechanically triturated into chyle-like shapes, and then filtered and centrifuged at $136 \mathrm{x} \mathrm{g}$ for $3 \mathrm{~min}$ at $4^{\circ} \mathrm{C}$. The supernatant was discarded and the cells were cultured in Dulbecco's modified Eagle's medium (DMEM). The filtered tissues were stirred in $10 \mathrm{X}$ volume of collagenase $(0.1 \%)$ in water at $37^{\circ} \mathrm{C}$ and sampled every $30 \mathrm{~min}$, three times. Between each step, the cells were centrifuged at $136 \mathrm{x} \mathrm{g}$ for $3 \mathrm{~min}$ at $4^{\circ} \mathrm{C}$, the supernatant was discarded and the cell suspension was resuspended in DMEM. Trypan blue staining was used to account for necrotic cells. The cells were counted under a CKX41SF inverted microscope (Olympus Corporation, Tokyo, Japan) and the cell density was adjusted to $1 \times 10^{6}$ cells $/ \mathrm{ml}$ for further use.

Detection of gastric smooth muscle cell apoptotic rates using flow cytometry. An Annexin V-FITC/propidium iodide (PI) flow cytometry kit (BD Biosciences, Franklin Lakes, NJ, USA) was used to detect cell apoptosis. Annexin V is regarded as a sensitive indicator of early apoptosis as it binds to phosphatidylserine on the cell membranes of early apoptotic cells, whereas PI stains cell nuclei red by readily crossing the cell membranes of late apoptotic and dead cells. Briefly, the cells were rinsed with phosphate-buffered saline twice and centrifuged at $243 \mathrm{x}$ g for $5 \mathrm{~min}$ at $4^{\circ} \mathrm{C}$, following which $1 \times 10^{5}$ cells were collected and suspended in $500 \mu \mathrm{l}$ binding buffer mixed with $5 \mu \mathrm{l}$ Annexin V-EGFP and $5 \mu \mathrm{l}$ PI. This mixture was incubated at room temperature in the dark for 5-15 min. Flow cytometry was used to observe cell apoptosis in the DM2W, DM4W and DM6W groups. The cells were divided into four quadrants, the abscissa was FITC-Annexin V, and the ordinate was PI. The upper right quadrant represented late apoptotic cells, the upper left quadrant represented necrotic cells, the lower left quadrant represented normal cells, and the lower right quadrant represented early apoptotic cells. Apoptotic rates were measured as the percentage of apoptotic cells to total cells.

Western blot assay of protein expression. The stomach muscle tissues were homogenized to extract total protein, the protein concentration was determined with a spectrophotometer at a wavelength of $596 \mathrm{~nm}$. The protein samples were boiled for 

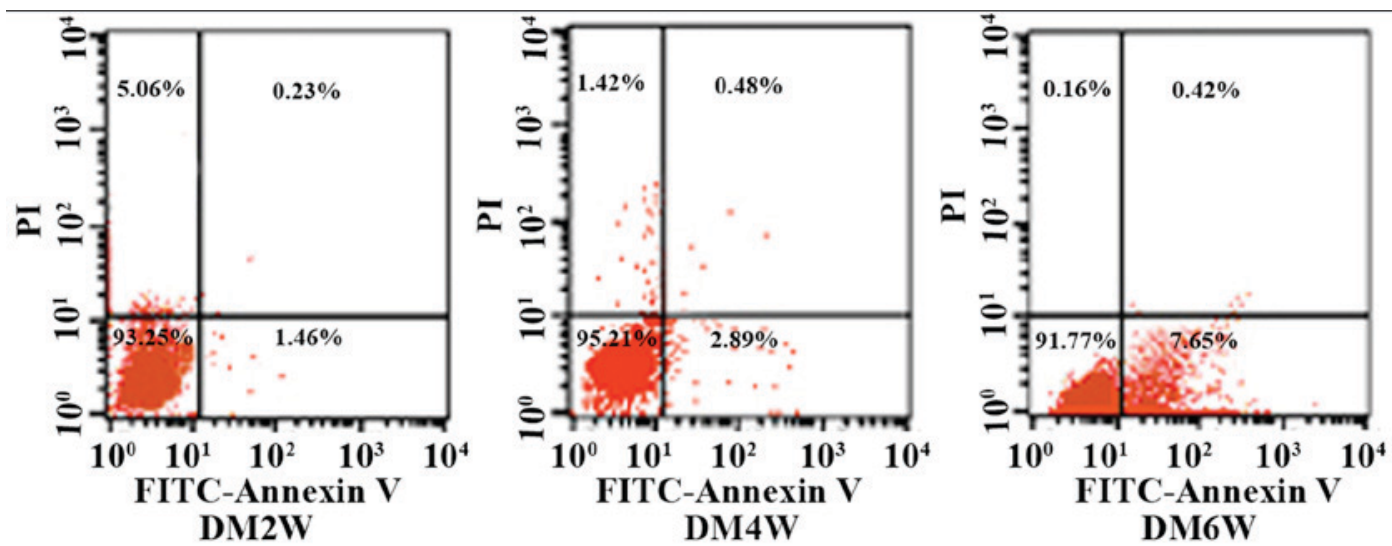

Figure 1. Cell apoptosis in the diabetes model at 2,4 and 6 weeks. The upper right quadrant represents late apoptotic cells, the upper left quadrant represents necrotic cells, the lower left quadrant represents normal cells and the lower right quadrant represents early apoptotic cells. DM2W, diabetic model 2 at weeks; DM4W, diabetic model at 4 weeks; DM6W, diabetic model at 6 weeks; PI, propidium iodide.

2 min and $40 \mu \mathrm{g}$ of proteins was loaded and separated via SDS-PAGE on a $10 \%$ gel. The protein was transferred onto a polyvinylidene difluoride (PVDF) membrane using a semi-dry transfer method. The sample was rinsed with $5 \%$ non-fat milk in TBS-T buffer [ $25 \mathrm{mmol} / \mathrm{l}$ Tris, $150 \mathrm{mmol} / \mathrm{l} \mathrm{NaCl}$ and $1 \%$ Tween 20 ( $\mathrm{pH} 7.5)$ ] and blocked on the PVDF membrane. The cells were then cultured with antibodies (all purchased from Cell Signaling Technology, Inc., Danvers, MA, USA) against PI3K (cat. no. 4263S, 1:1,000), p-AKT (Ser473; cat. no. 4060S; 1:500), p-mTOR (Ser2481; cat. no. 2974S; 1:1,000), p-AMPK (Thr172; cat. no. 2531S; 1:1,000), p-TSC-2 (Thr1462; cat. no. 3617S; 1:1,000), p-p70S6K (Thr389; cat. no. 9234S; 1:500), P-4E-BP1 (Thr37/46; cat. no. 2855S; 1:500), and $\beta$-actin (1:500) at $4^{\circ} \mathrm{C}$ overnight. The cells were washed with $0.01 \mathrm{M}$ PBS and incubated with horseradish peroxidase-conjugated goat anti-rabbit IgG (1:1,000; Sigma-Aldrich; Merck KGaA) at room temperature for $1 \mathrm{~h}$. A gel imaging analysis system was used to acquire and analyze images; $\beta$-actin (cat. no. A5316-.2ML; 1:500; Sigma-Aldrich; Merck KGaA) served as a reference against which protein content was calculated.

Statistical analysis. SPSS 19.0 software (IBM SPSS, Armonk, NY, USA) was used for statistical analysis. Measurement data are expressed as the means \pm standard deviation. Differences between groups were compared using an independent sample t-test and one-way analysis of variance. $\mathrm{P}<0.05$ was considered to indicate a statistically significant difference.

\section{Results}

Comparison of gastric residual pigment ratios. The results of the present study indicated the gastric residual pigment ratio was $40.24 \pm 2.15$ in the NC group and 50.65 \pm 3.31 in the DM6W group $(\mathrm{n}=10 ; \mathrm{P}<0.05$; Table I), suggesting that the diabetic rats exhibited symptoms of stomach paresis at 6 weeks, with symptoms worsening with time.

Comparison of gastric smooth muscle cell apoptotic rates. The results of the present study showed that apoptotic gastric smooth muscle cells were apparent in each diabetic model group, and the rates of apoptosis increased as the disease

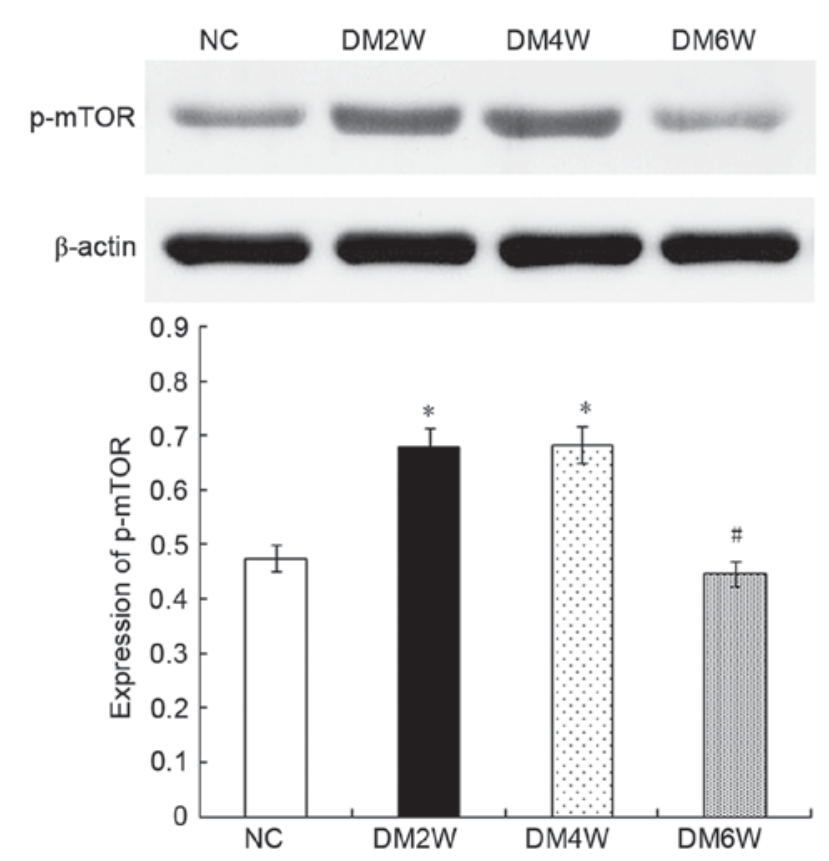

Figure 2. Expression of p-mTOR in each group. * $\mathrm{P}<0.01$, vs. NC group; ${ }^{\#} \mathrm{P}<0.01$, vs. DM4W group. p-mTOR, phosphorylated, mammalian target of rapamycin; NC, normal control; DM2W, diabetic model at 2 weeks; DM4W, diabetic model at 4 weeks; diabetic model at 6 weeks.

progressed. The rates of apoptosis were $1.54 \pm 0.46$ in the DM2 W group, $2.75 \pm 0.54$ in the DM4 W group, and 7.48 \pm 0.36 in the DM6 W group $(\mathrm{n}=10 ; \mathrm{P}<0.05$; Table I; Fig. 1).

Expression of key PI3K-AKT-mTOR and AMPKOmTOR pathway proteins in rat gastric muscle

Relative protein expression of $p$-mTOR. The expression of p-mTOR was $0.48 \pm 0.03$ in the NC group, which was lower than the level observed in the DM2W $(0.67 \pm 0.04)$ and DM4W $(0.68 \pm 0.03)$ groups $(n=10 ; \mathrm{P}<0.01)$. Compared with the DM4W group, the DM6W group showed lower levels of p-mTOR $(0.45 \pm 0.02 ; n=10 ; \mathrm{P}<0.01)$. As shown in Fig. 2, no significant difference was observed between the $\mathrm{NC}$ and DM6W groups. 

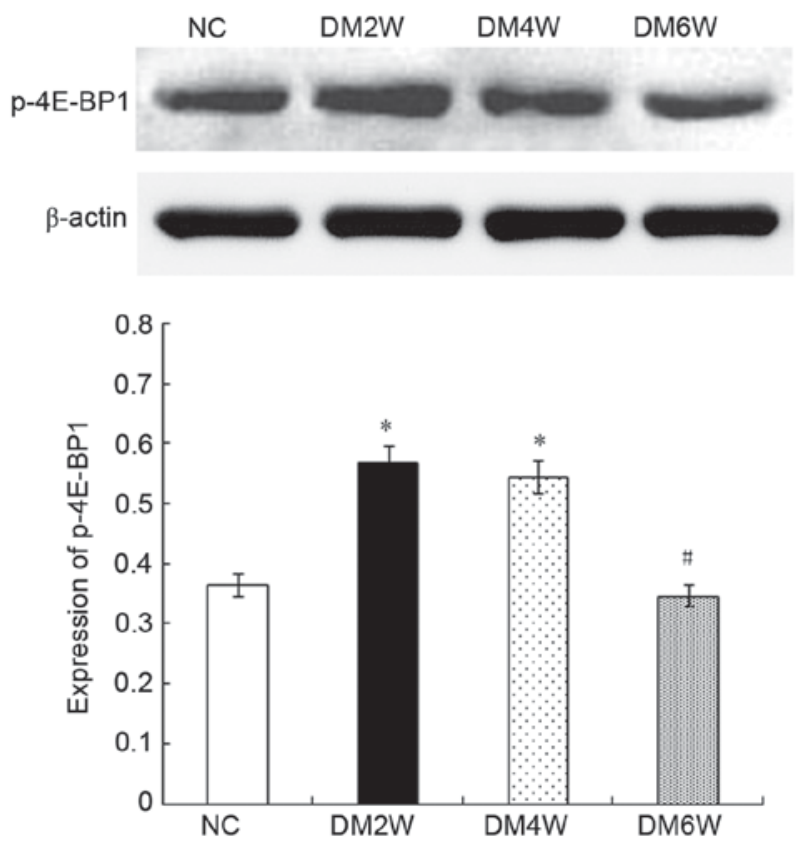

Figure 3. Expression of $\mathrm{P}-4 \mathrm{E}-\mathrm{BP} 1$ in each group. ${ }^{*} \mathrm{P}<0.01$, vs. normal control group; " $\mathrm{P}<0.01$, vs. DM4W group. P-4E-BP1, phosphorylated eukaryotic translation initiation factor 4-binding protein 1; NC, normal control; DM2W, diabetic model at 2 weeks; DM4W, diabetic model at 4 weeks; diabetic model at 6 weeks.
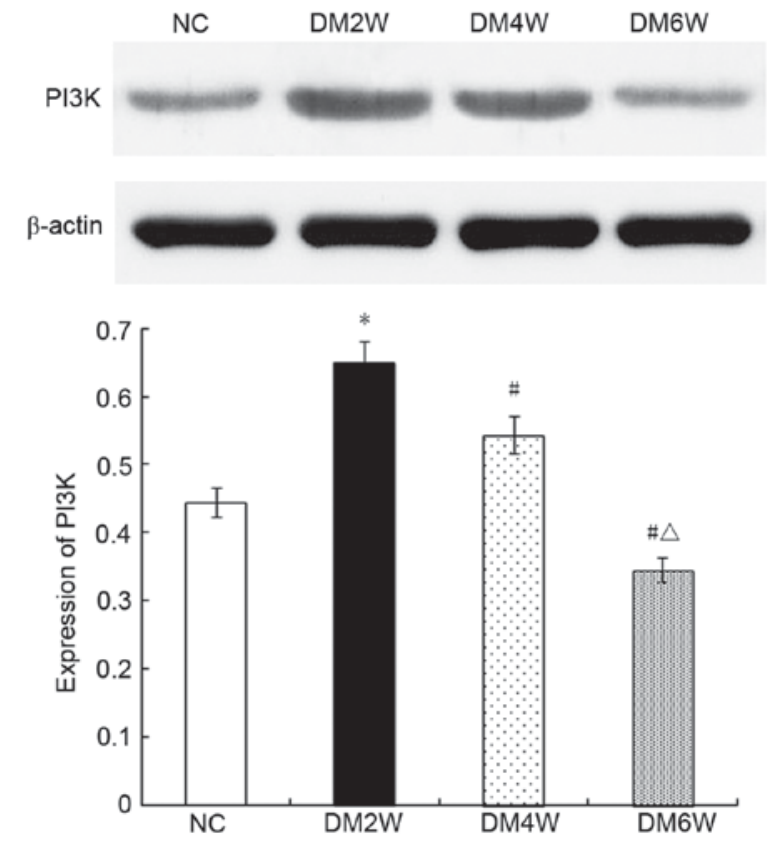

Figure 5. Expression of $\mathrm{PI} 3 \mathrm{~K}$ in each group. ${ }^{*} \mathrm{P}<0.01,{ }^{\#} \mathrm{P}<0.05$, vs. normal control group; ${ }^{\Delta} \mathrm{P}<0.01$, vs. DM4W group. $\mathrm{PI} 3 \mathrm{~K}$, phosphoinositide-3-kinase; NC, normal control; DM2W, diabetic model at 2 weeks; DM4W, diabetic model at 4 weeks; diabetic model at 6 weeks.
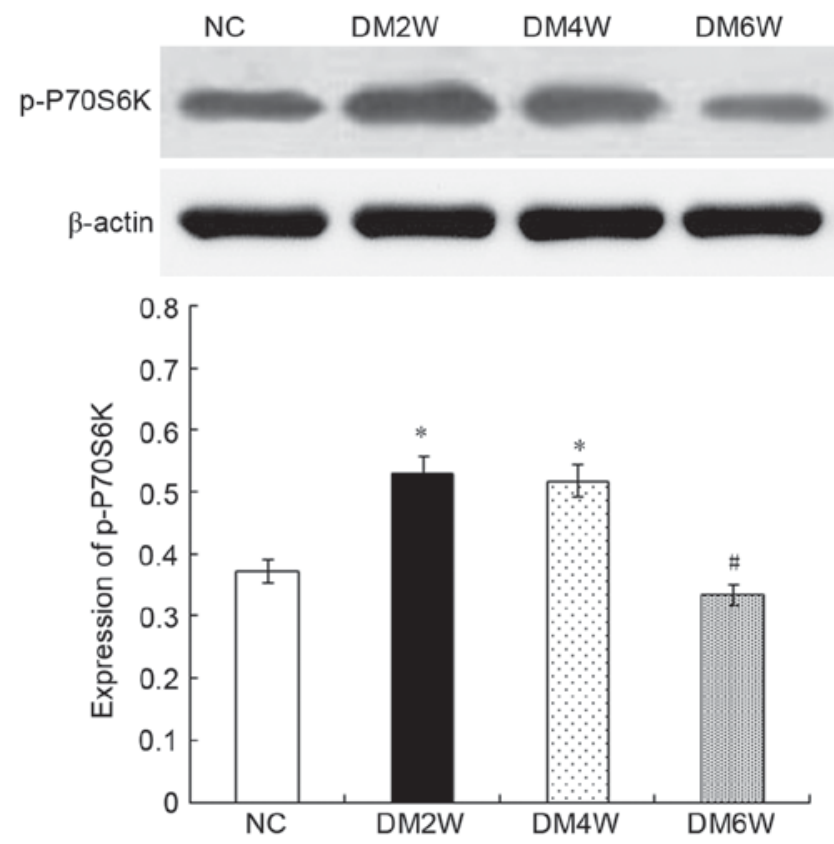

Figure 4. Expression of p-P70S6K in each group. ${ }^{*} \mathrm{P}<0.01$, vs. normal control group; ${ }^{\text {P }}<0.01$, vs. DM4W group. p-P70S6K, phosphorylated p70 ribosomal S6 kinase; NC, normal control; DM2W, diabetic model at 2 weeks; DM4W, diabetic model at 4 weeks; diabetic model at 6 weeks.

Relative protein expression of $P-4 E-B P 1$. Compared with the $\mathrm{NC}$ group $(0.36 \pm 0.05)$, the expression levels of P-4E-BP1 were higher in the DM2W $(0.57 \pm 0.03)$ and DM4W $(0.54 \pm 0.04)$ groups $(\mathrm{n}=10 ; \mathrm{P}<0.01)$. However, compared with the DM4W group, the DM6W group exhibited a lower expression level of $\mathrm{P}-4 \mathrm{E}-\mathrm{BP} 1(0.35 \pm 0.02 ; \mathrm{n}=10 ; \mathrm{P}<0.01)$. No significant difference was observed between the NC and DM6W groups, as shown in Fig. 3.
Relative protein expression of $p$-P70S6K. Compared with the NC group $(0.37 \pm 0.04)$, the expression levels of p-P70S6K were higher in the DM2W $(0.53 \pm 0.02)$ and DM4W $(0.52 \pm 0.02)$ groups $(\mathrm{n}=10 ; \mathrm{P}<0.01)$. The DM6W group $(0.34 \pm 0.02)$ exhibited a decreased expression level of p-P70S6K compared with the DM4W group $(n=10 ; \mathrm{P}<0.01)$. As shown in Fig. 4, no significant difference was observed between the $\mathrm{NC}$ and DM6W groups.

Relative protein expression of PI3K. Compared with the NC group $(0.44 \pm 0.03)$, the expression levels of PI3K were higher in the DM2W $(0.65 \pm 0.03)$ and DM4W $(0.54 \pm 0.06)$ groups $(\mathrm{n}=10 ; \mathrm{P}<0.01)$, whereas the DM6W group exhibited lower expression $(0.35 \pm 0.05 ; \mathrm{n}=10 ; \mathrm{P}<0.01)$. The expression levels were also lower in the DM4W and DM6W groups $(n=10$; $\mathrm{P}<0.01$ ), as shown in Fig. 5.

Relative protein expression of $p$-AKT. The expression levels of p-AKT were higher in the DM2W $(0.75 \pm 0.02)$ and DM4W $(0.73 \pm 0.03)$ groups, compared with that in the NC group $(0.53 \pm 0.02 ; \mathrm{n}=10 ; \mathrm{P}<0.01)$. By contrast, the DM6W group exhibited lower expression $(0.44 \pm 0.02 ; n=10 ; \mathrm{P}<0.01)$. As shown in Fig. 6, the expression levels were significantly lower in the DM4W and DM6W groups, compared with that in the DM2W group $(n=10 ; \mathrm{P}<0.01)$.

Relative protein expression of $p$-AMPK. Compared with the $\mathrm{NC}$ group $(0.34 \pm 0.04)$, the expression levels of p-AMPK were higher in the DM2W $(0.58 \pm 0.05)$, DM4W $(0.70 \pm 0.05)$ and DM6W $(0.69 \pm 0.05)$ groups $(n=10 ; \mathrm{P}<0.01)$. The expression levels were increased in the DM4W and DM6W groups, compared with that in the DM2W group $(\mathrm{n}=10 ; \mathrm{P}<0.01)$; however, no significant difference was observed between the DM4W and DM6W groups, as shown in Fig. 7. 

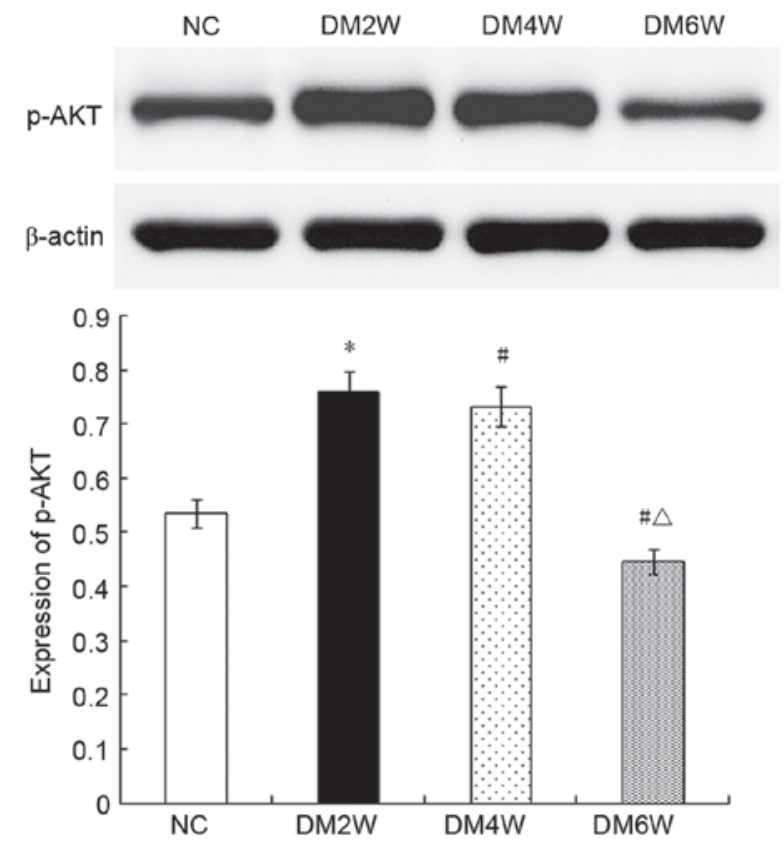

Figure 6. Expression of p-AKT in each group. ${ }^{*} \mathrm{P}<0.01$ and ${ }^{~} \mathrm{P}<0.05$, vs normal control group; ${ }^{\triangle} \mathrm{P}<0.01$, vs. DM4W group. $\mathrm{p}$-AKT, phosphorylated protein kinase B; NC, normal control; DM2W, diabetic model at 2 weeks; DM4W, diabetic model at 4 weeks; diabetic model at 6 weeks.
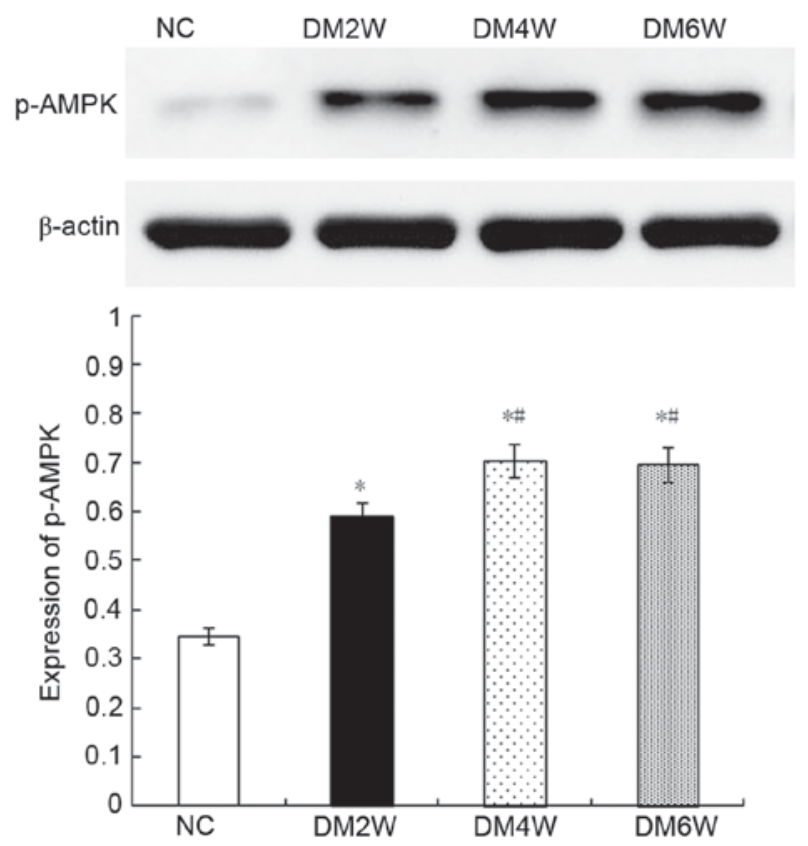

Figure 7. Expression of p-AMPK in each group. ${ }^{*} \mathrm{P}<0.01$, vs. normal control group; ${ }^{~} \mathrm{P}<0.05$, vs. DM2W group. p-AMPK, phosphorylated 5' adenosine monophosphate-activated protein kinase; NC, normal control; DM2W, diabetic model at 2 weeks; DM4W, diabetic model at 4 weeks; diabetic model at 6 weeks.

Relative protein expression of $p$-TCS-2. Compared with the $\mathrm{NC}$ group (0.32 \pm 0.02$)$, the expression levels of p-TCS-2 were higher in the DM2W $(0.60 \pm 0.03)$, DM4W $(0.72 \pm 0.02)$ and DM6W $(0.73 \pm 0.04)$ groups $(\mathrm{n}=10 ; \mathrm{P}<0.01)$. Compared with the DM2W group, the expression was increased in the DM4W group $(\mathrm{n}=10 ; \mathrm{P}<0.01)$. No significant difference was observed between the DM4W and DM6W groups, as shown in Fig. 8.
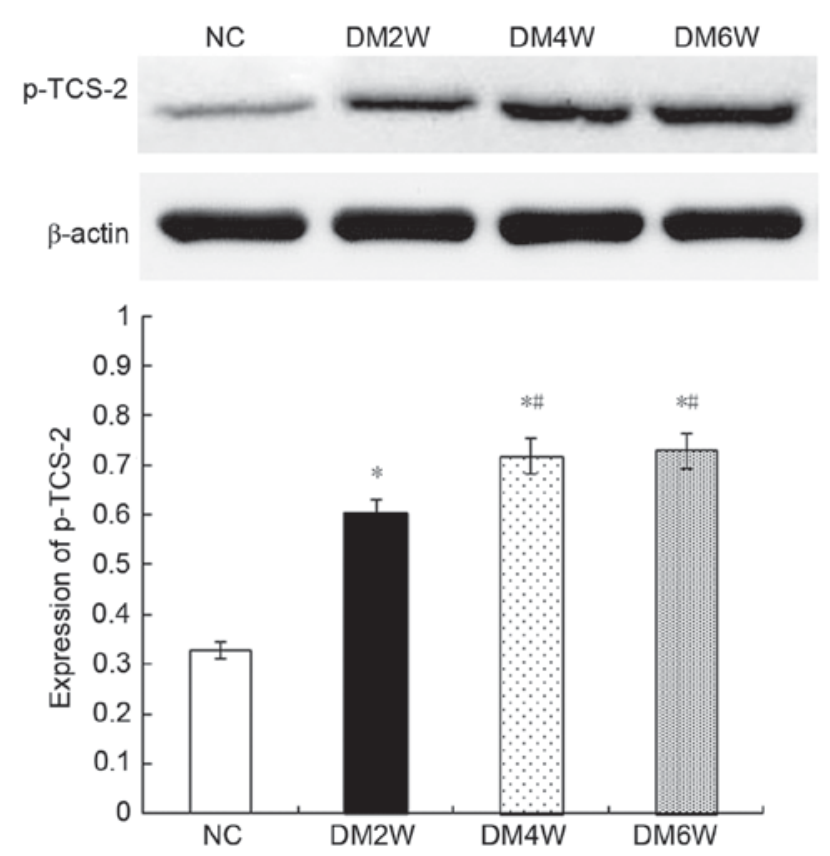

Figure 8. Expression of p-TCS-2 in each group. ${ }^{*} \mathrm{P}<0.01$, vs. normal control group; " $\mathrm{P}<0.05$, vs. DM2W group. p-TCS-2, phosphorylated tuberous sclerosis complex 2; NC, normal control; DM2W, diabetic model at 2 weeks; DM4W, diabetic model at 4 weeks; diabetic model at 6 weeks.

\section{Discussion}

Diabetic gastroparesis is a complex pathophysiological process (15). Investigations on the pathogenesis of diabetic gastroparesis predominantly focus on high blood sugar-induced neuropathy, stomach hormone secretion disorder, stress, and certain microvascular diseases $(16,17)$, whereas few report on apoptosis and its regulatory pathways. Apoptosis can be induced and controlled by a variety of cytokines (18). As each tissue or cell can react differently to different factors, each cytokine can exhibit different biological effects $(19,20)$. Apoptosis is triggered by anti- and pro-apoptotic factors, including the PI3K-AKT-mTOR pathway, which inhibits cell apoptosis. Under normal circumstances, growth factors, including insulin, insulin-like growth factor and epidermal growth factor, induce PI3K-AKT-mTOR activation via their respective receptors (21). By contrast, the AMPK-mTOR pathway has a pro-apoptotic role. Under conditions of stress, including ischemia, hypoxia or nutrient deficiency, the AMPK-mTOR pathway is activated to relieve stress and maintain normal body metabolism (22).

Hyperglycemia, a characteristic clinical manifestation of diabetes mellitus, forms the basis of the majority of diabetes complications. It enables cellular oxidative stress and metabolic disorders, resulting in hypoxia, decreased amino acid levels, low adenosine triphosphate and/or high adenosine monophosphate. Previous studies have found that these changes may alter the biological effects of mTOR through the AMPK or PI3K-AKT pathway to regulate apoptosis $(23,24)$. To investigate the significance of apoptosis in diabetic gastroparesis, in addition to changes in PI3K-AKT-mTOR and AMPK-mTOR signaling, the present study established a diabetic rat model to determine gastric residual pigment ratios and the timing of the occurrence of diabetic gastroparesis. It was found that 
diabetic gastroparesis was present in diabetic rats at 6 weeks, with the diabetic rats beginning to show symptoms of gastroparesis at 6 weeks. This differs from the findings of a previous study (25), which may be associated with differences between individual animals and feeding conditions. Following establishment of the diabetic gastroparesis model, it was found that the apoptotic rates of the gastric smooth muscle cells during diabetic gastroparesis gradually increased, with significance at 6 weeks. As apoptosis is a form of programmed cell death, increased apoptotic rates directly result in the reduction of normal functional cells, thereby prolonging gastric emptying. This evidence indicated that increased apoptosis may be an important cause of diabetic gastroparesis, consistent with the findings of previous studies (3).

mTOR is a protein factor regulated by the PI3K-AKT-mTOR pathway and the AMPK-mTOR pathway. Although the primary activity of mTOR is the phosphorylation of mTOR, its regulation during apoptosis is achieved by the phosphorylation of downstream 4E-BP1 and p70S6K (11). Therefore, the expression levels of p-mTOR, P-4E-BP1 and p-p70S6K reflect the effect of mTOR on apoptosis. In the present study, the results of the western blot analysis revealed increased expression levels of p-mTOR, P-4E-BP1 and p-p70S6K when diabetic gastroparesis began to occur, and decreased expression following its establishment, indicating the involvement of mTOR and its downstream factors in the initial occurrence of diabetic gastroparesis. The initial increases in mTOR activity and downstream factors were associated with the inhibition of cell apoptosis. Of note, the rates of cell apoptosis increased continuously during diabetic gastroparesis, indicating that the anti-apoptotic role of mTOR was not dominant.

PI3K-AKT and AMPK are positive and negative regulatory factors of the activity of mTOR, respectively. PI3K, p-AKT, p-AMPK and p-TCS-2 are functional proteins involved in these two pathways, therefore, their expression level directly affects the activity of the pathways. Western blot analysis was performed to investigate the regulation of mTOR activity by the PI3K-AKT and AMPK pathways during diabetic gastroparesis, which revealed similar changes in the expression of PI3K and p-AKT, which initially increased and then decreased. p-AMPK and p-TCS-2 also exhibited similar expression patterns, which initially increased and were then maintained. Collectively, these results indicated that the PI3K-AKT and AMPK pathways were involved in the occurrence of diabetic gastroparesis, and the mechanism may be associated with the mTOR-mediated regulation of apoptosis.

During the early stage of diabetic gastroparesis, the expression of proteins in the PI3K-AKT pathway increased, which promoted the phosphorylation of mTOR and inhibited anti-apoptotic activity. A potential reason for this is that, in response to initial stimulation with high glucose, gastric smooth muscle cells compensate by increasing upstream growth factors in the PI3K-AKT pathway by autocrine or paracrine mechanisms to maintain cellular function, thus activating the PI3K-AKT pathway. When diabetic gastroparesis results from continuous stimulation by hyperglycemia, the expression of PI3K-AKT upstream growth factors was decreased, therefore, the activity of PI3K-AKT was decreased. The changes in AMPK differed from those of PI3K-AKT, as the expression of functional proteins continuously increased over the duration of the diabetic gastroparesis process, indicating activation of its pro-apoptotic role. This is consistent with the observed increases in apoptotic rates when diabetic gastroparesis occurred. Of note, the activity of mTOR downstream of AMPK initially increased and then was inhibited, suggesting that AMPK was inferior to PI3K-AKT in regulating the activity of mTOR. During the early stage of diabetic gastroparesis, the PI3K-AKT-mediated activation of mTOR increased, which weakened the AMPK-induced inhibition of mTOR activity. With prolonged duration, the activity of PI3K-AKT decreased and that of AMPK increased. Therefore, when diabetic gastroparesis was established, a decrease in the activity of mTOR occurred.

In conclusion, the present study confirmed that cell apoptosis was important in the occurrence of diabetic gastroparesis. During this process, the PI3K-AKT-mTOR and AMPK-mTOR pathways were activated, but were unable to regulate apoptosis. Further investigations aim to focus on how the activated PI3K-AKT-mTOR and AMPK-mTOR pathways are involved in establishing diabetic gastroparesis.

\section{Acknowledgements}

This study was financially supported by grants from the National Natural Science Foundation of China (grant nos. 81360070 and 81560142 ).

\section{References}

1. Zhao J, Frøkjaer JB, Drewes AM and Ejskjaer N: Upper gastrointestinal sensory-motor dysfunction in diabetes mellitus. World J Gastroenterol 12: 2846-2857, 2006.

2. Jin QH, Shen HX, Wang H, Shou QY and Liu Q: Curcumin improves expression of SCF/c-kit through attenuating oxidative stress and NF- $\kappa$ B activation in gastric tissues of diabetic gastroparesis rats. Diabetol Metab Syndr 5: 12, 2013.

3. Chen X, Fu XS, Li CP and Zhao HX: ER stress and ER stress-induced apoptosis are activated in gastric SMCs in diabetic rats. World J Gastroenterol 20: 8260-8267, 2014.

4. Lu D, Qian J, Li W, Feng Q, Pan S and Zhang S: $\beta$-hydroxyisovaleryl-shikonin induces human cervical cancer cell apoptosis via PI3K/AKT/mTOR signaling. Oncol Lett 10: 3434-3442, 2015.

5. Cui H, Wu S, Shang Y, Li Z, Chen M, Li F and Wang C: Pleurotus nebrodensis polysaccharide(PN50G) evokes A549 cell apoptosis by the ROS/AMPK/PI3K/AKT/mTOR pathway to suppress tumor growth. Food Funct 7: 1616-1627, 2016.

6. Guo S, Yao Q, Ke Z, Chen H, Wu J and Liu C: Resveratrol attenuates high glucose-induced oxidative stress and cardiomyocyte apoptosis through AMPK. Mol Cell Endocrinol 412: 85-94, 2015.

7. Han G, Gong H, Wang Y, Guo S and Liu K: AMPK/mTOR-mediated inhibition of survivin partly contributes to metformin-induced apoptosis in human gastric cancer cell. Cancer Biol Ther 16: 77-87, 2015.

8. Zhou Y, Liang X, Chang H, Shu F, Wu Y, Zhang T, Fu Y, Zhang Q, Zhu JD and Mi M: Ampelopsin-induced autophagy protects breast cancer cells from apoptosis through Akt-mTOR pathway via endoplasmic reticulum stress. Cancer Sci 105: 1279-1287, 2014

9. Wani ZA, Guru SK, Rao AV, Sharma S, Mahajan G, Behl A, Kumar A, Sharma PR, Kamal A, Bhushan S and Mondhe DM: A novel quinazolinone chalcone derivative induces mitochondrial dependent apoptosis and inhibits PI3K/Akt/mTOR signaling pathway in human colon cancer HCT-116 cells. Food Chem Toxicol 87: 1-11, 2016.

10. Yang J, Cheng D,Zhou S,Zhu B,Hu T and Yang Q: Overexpression of X-Box binding protein 1 (XBP1) correlates to poor prognosis and up-regulation of PI3K/mTOR in human osteosarcoma. Int J Mol Sci 16: 28635-28646, 2015. 
11. Zi D, Zhou ZW, Yang YJ, Huang L, Zhou ZL, He SM, He ZX and Zhou SF: Danusertib induces apoptosis, cell cycle arrest, and autophagy but inhibits epithelial to mesenchymal transition involving PI3K/Akt/mTOR Signaling Pathway in Human Ovarian Cancer Cells. Int J Mol Sci 16: 27228-27251, 2015.

12. Xu DY, Liu L, Cai YL, Li XL, Qiu ZX, Jin Z and Xu WX: Natriuretic peptide-dependent cGMP signal pathway potentiated the relaxation of gastric smooth muscle in streptozotocin-induced diabetic rats. Dig Dis Sci 55: 589-595, 2010.

13. Cai YL, Xu DY, Li XL, Qiu ZX, Jin Z and Xu WX: C-type natriuretic-peptide-potentiated relaxation response of gastric smooth muscle in streptozotocin-induced diabetic rats. World J Gastroenterol 15: 2125-2131, 2009.

14. Asano T, Aida S, Suemasu S and Mizushima T: Anethole restores delayed gastric emptying and impaired gastric accommodation in rodents. Biochem Biophys Res Commun 472: 125-130, 2016

15. Tack J, Carbone F and Rotondo A: Gastroparesis. Curr Opin Gastroenterol 31: 499-505, 2015.

16. Singla R, Homko C, Schey R and Parkman HP: Diabetes-related autoantibodies in diabetic gastroparesis. Dig Dis Sci 60 $1733-1737,2015$

17. King RJ, Harrison L, Gilbey SG, Santhakumar A, Wyatt J, Jones R and Bodansky HJ: Diabetic hepatosclerosis: Another diabetes microvascular complication? Diabet Med 33: e5-e7, 2016.

18. Goldar S, Khaniani MS, Derakhshan SM and Baradaran B: Molecular mechanisms of apoptosis and roles in cancer development and treatment. Asian Pac J Cancer Prev 16: 2129-2144, 2015 .
19. Childs BG,Baker DJ, Kirkland JL,Campisi J and van Deursen JM: Senescence and apoptosis: Dueling or complementary cell fates? EMBO Rep 15: 1139-1153, 2014

20. Sankari SL, Babu NA, Rajesh E and Kasthuri M: Apoptosis in immune-mediated diseases. J Pharm Bioallied Sci 7 (Suppl 1): S200-S202, 2015.

21. Vanhaesebroeck B, Stephens L and Hawkins P: PI3K signalling The path to discovery and understanding. Nat Rev Mol Cell Biol 13: 195-203, 2012.

22. Jin Y, Bai Y, Ni H, Qiang L, Ye L, Shan Y and Zhou M: Ac tivation of autophagy through calcium-dependent AMPK/mTOR and PKC $\theta$ pathway causes activation of rat hepatic stellate cells under hypoxic stress. FEBS Lett 590: 672-682, 2016

23. Kumar S, Guru SK, Pathania AS, Manda S, Kumar A, Bharate SB, Vishwakarma RA, Malik F and Bhushan S: Fascaplysin induces caspase mediated crosstalk between apoptosis and autophagy through the inhibition of PI3K/AKT/mTOR signaling cascade in human leukemia HL-60 cells. J Cell Biochem 116: 985-997, 2015.

24. Ma MQ, Thapalia BA and Lin XH: A 6 hour therapeutic window, optimal for interventions targeting AMPK synergism and apoptosis antagonism, for cardioprotection against myocardial ischemic injury: An experimental study on rats. Am J Cardiovasc Dis 5: 63-71, 2015

25. Jin QH, Shen HX, Wang H, Shou QY and Liu Q: Curcumin improves expression of $\mathrm{SCF} / \mathrm{c}$-kit through attenuating oxidative stress and NF- $\kappa \mathrm{B}$ activation in gastric tissues of diabetic gastroparesis rats. Diabetol Metab Syndr 5: 12, 2013. 